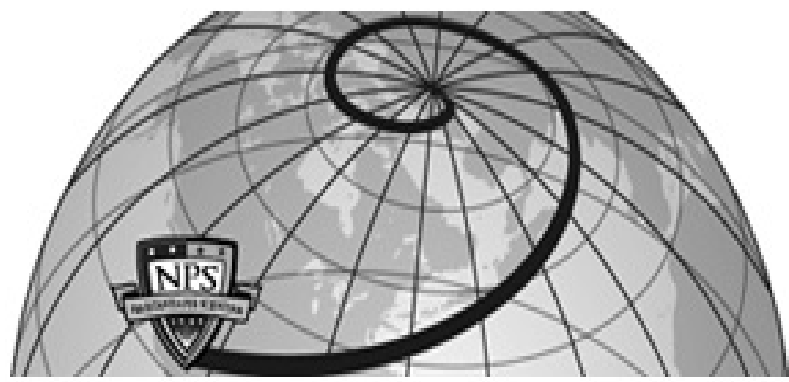

Calhoun: The NPS Institutional Archive DSpace Repository

Incorporating Realistic Acoustic Propagation Models in Simulation of Underwater Acoustic Networks: A Statistical Approach

Gibson, J.; Diaz-Gonzalez, L.; Xie, Geoffrey

https://hdl.handle.net/10945/34779

This publication is a work of the U.S. Government as defined in Title 17, United States Code, Section 101. Copyright protection is not available for this work in the United States.

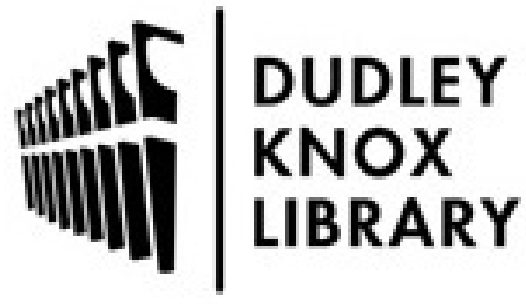

http://www.nps.edu/library
Calhoun is the Naval Postgraduate School's public access digital repository for research materials and institutional publications created by the NPS community. Calhoun is named for Professor of Mathematics Guy K. Calhoun, NPS's first appointed -- and published -- scholarly author.

Dudley Knox Library / Naval Postgraduate School 411 Dyer Road / 1 University Circle Monterey, California USA 93943 


\title{
Incorporating Realistic Acoustic Propagation Models in Simulation of Underwater Acoustic Networks: A Statistical Approach
}

\author{
Geoffrey Xie \\ John Gibson Leopoldo Diaz-Gonzalez \\ Naval Postgraduate School \\ Monterey, CA 93943 USA
}

Abstract- The development of protocols to advance the state of the art in Underwater Acoustic Networks (UANs) relies on the use of computer simulations to analyze protocol performance. It is typical for designers to abstract away much of the detail of the physical environment in order to simplify the development of the simulation and ensure the simulation runtime performance is reasonable. The validity of the simulation results becomes questionable. There are, though, very high fidelity models developed by acoustic engineers and physicists for predicting acoustic propagation characteristics. In addition to these models, empirical data collections have been generated for many geographic regions of interest to UAN planners. However, incorporating these engineering and physics models or data collections into a network simulation is problematic, as the models are computationally complex and the data sets are not directly usable for acoustic signal propagation characterization.

This paper presents a statistical method for developing a computationally efficient and simulation friendly approximation of a physics model of path loss. This method may also be used to adapt empirical data sets for use in network simulation in the same manner. The method was applied to the output of the Monterey-Miami Parabolic Equation model to assess its impact on the runtime performance of an OPNET-based simulation. Results of that simulation are compared to results from a previous OPNET simulation that simply used distance to determine reception. The simulation results confirm the incorporation of the MMPE approximation does not noticeably impact the runtime performance of the simulation. Anecdotally, the

simulation confirms earlier results indicating that contentionbased access controls without collision avoidance techniques may outperform the typical access technique adapted from wireless radio network and employing collision avoidance, even in the high load regime, contrary to conventional wisdom.

\section{INTRODUCTION}

Current underwater acoustic networks (UANs) predominantly borrow networking protocols developed for the radio frequency (RF) environment, e.g., 802.11 media access control for collision avoidance. Recently, some network protocol designers have raised serious doubts regarding the effectiveness of the RF protocols in the underwater acoustic environment, where the bandwidth is several magnitudes smaller and the signal propagation is several magnitudes slower, especially for small data packets [1-3]. Evaluation of recent experimental data supports that concern, where it may be questioned whether or not the collision avoidance scheme is actually detrimental to network performance [4]. Meanwhile, typical UAN experimentation is still focused on setting up and testing primitive network functions with artificial (and often extremely light) traffic patterns and limited node density. Protocol designers have mostly turned to computer simulation for validating their forward looking ideas, such as node clustering $[5,6]$. However, the utility of existing UAN simulation tools is questionable as these tools suffer from a major weakness: there is no detailed modeling of the physical layer properties suitable for use in network simulations, even though the underwater acoustic channel environment is much harsher than RF and, therefore, is more likely to cause performance problems in the upper layer protocols. This lack of robust simulation-capable propagation loss models generally forces network protocol designers to use simplifying assumptions to address propagation losses.

Two such modeling efforts include the design and simulation of an underwater acoustic LAN by Sozer, et al. [6] and an analysis of medium access control scheme for UANs by Coelho $[7,8]$. In the former the propagation loss is modeled using a form of the Thorp equation where loss is determined by frequency, range and an added static background noise level and random fading component $[9,10]$. The latter simplifies the loss even further by using only a static range value. While neither of these models was intended to explicitly model the physical environment, the harshness of that environment to uniform signal propagation can lead to speculation as to the validity of the results achieved. In particular, the Sozer model focused on demonstrating a packet forwarding capability through a sensor network using collision avoidance and the Coelho model focused on comparing the relative performance of collision avoidance and Aloha derived protocols in the excessive delay constrained environment. These models highlight the dichotomy between models developed by acoustic engineers and physicist and those suitable for time-constrained event-driven simulations. Fig. 1 portrays this situation, where acoustic engineering models have both high complexity and high fidelity, whereas computer simulation models accept lower fidelity in order to ensure computational feasibility.

The results of the acoustic engineering models, primarily based on ray tracing or parabolic equations, are used to guide the development of acoustic modems for developing underwater 


\section{Report Documentation Page}

Form Approved

OMB No. 0704-0188

Public reporting burden for the collection of information is estimated to average 1 hour per response, including the time for reviewing instructions, searching existing data sources, gathering and maintaining the data needed, and completing and reviewing the collection of information. Send comments regarding this burden estimate or any other aspect of this collection of information,

including suggestions for reducing this burden, to Washington Headquarters Services, Directorate for Information Operations and Reports, 1215 Jefferson Davis Highway, Suite 1204, Arlington

VA 22202-4302. Respondents should be aware that notwithstanding any other provision of law, no person shall be subject to a penalty for failing to comply with a collection of information if it

does not display a currently valid OMB control number.

\begin{tabular}{l|l|l} 
1. REPORT DATE & 2. REPORT TYPE & 3. DATES COVERED \\
SEP 2006 & N/A &
\end{tabular}

12. DISTRIBUTION/AVAILABILITY STATEMENT

Approved for public release, distribution unlimited

13. SUPPLEMENTARY NOTES

In Proceedings of MTS/IEEE Oceans Conference, Boston, September 2006, The original document contains color images.

14. ABSTRACT

see report

15. SUBJECT TERMS

16. SECURITY CLASSIFICATION OF:

a. REPORT

unclassified b. ABSTRACT unclassified c. THIS PAGE unclassified
17. LIMITATION OF ABSTRACT

SAR

\begin{tabular}{l|l}
$\begin{array}{c}\text { 18. NUMBER } \\
\text { OF PAGES }\end{array}$ & 19a. NAME OF \\
$\mathbf{9}$ & RESPONSIBLE PERSON \\
&
\end{tabular}




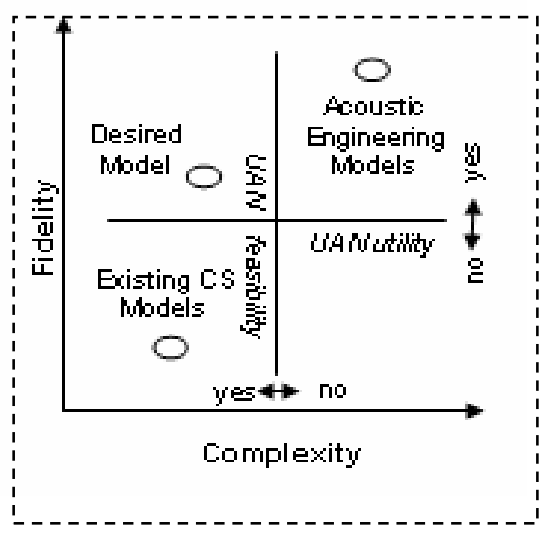

Figure 1: Acoustic Model Spectrum

acoustic communications systems and to characterize the expected sound profile for various aquatic environments. These tend to be less simulation friendly, as they often require special processing in order to produce extractable results. Such postprocessing makes it difficult to incorporate them in off-the-shelf simulations. In particular, parabolic equations use split-step, Fast Fourier Transform to iteratively determine the signal loss as referenced from a single source point. Two such model are the Navy Standard Parabolic Equation Model, developed by the Naval Research Laboratory (NRL) and implemented as the standard for operation acoustic modeling [11], and the Monterey-Miami Parabolic Equation (MMPE) developed by Kevin Smith and Fred Tappert $[12,13]$. These models provide very robust estimates of the signal loss for specific channel contours, as they may be configured to consider both bottom contours and surface activity. As such the values generated are specific to the geographic area or contour specified as a model input. In contrast, the models of Sozer and Coelho, use propagation loss models that are nonspecific as to the modeled environment, thus the results are not necessarily reflective of a particular target environment.

UAN models, such as Sozer's and Coelho's, focus on the transfer of messages between nodes comprising the network, placing less emphasis on the specifics of the underlying physical channel, by employing simplifying assumptions, such as propagation loss consistency across the duration of a packet and bi-directionally equivalent links. Without simulation-viable propagation models such assumptions are necessary to produce responsive simulations. Thus, for such simulations the physical model is effectively split into two parts: the simplified propagation estimate and the performance characteristics of a particular hardware suite being studied. This allows a network model to consider the source level, transducer gain, and receiver gain as reception parameters while assuming a simplified propagation loss model, in cases where the performance of a particular modem is of interest. Other simulations are intended to provide general trends in the performance of the protocol being analyzed and assumptions about the physical layer, while making the simulation responsive, may mask real phenomena which would otherwise adversely impact the protocol performance.
Including topology-dependent constraints into an UAN performance model in order to relax the simplifying assumptions that may limit the fidelity of UAN models is nontrivial, as the models used to predict acoustic propagation tend to be computationally complex and do not lend themselves to the repetitive nature of loss calculations necessary to support predictive analysis of message-based systems. For example, a single run of the MMPE on 64-bit laptop can take up 30 minutes, at the frequency of interest, and must be run for each potential message recipient [14]. Thus, to model a single message that flows through a relatively small network of 6 hops, where each hop neighborhood consists of only three nodes, would require 18 runs of the MMPE resulting in a processing delay of up to 9 hours. Such a procedure would not support extended network performance analysis. Further, the MMPE required postprocessing of the data using Matlab, further obfuscates its utility in network simulations. What is needed is a simulation "friendly" method that can take into account the contour of the target environment, often available from public and private repositories such as the NRL or NOAA, for areas of common interest and is computationally viable on standard desktop computer systems.

To address the bi-directional connectivity and consistent propagation loss assumptions, a physical layer model is required that can provide reasonable estimates of the propagation character of the water channel without significantly impacting the duration of each simulation run. As multiple runs must be accomplished to make supportable claims based on the results of those simulation runs, it is imperative that the duration of each run be constrained. This paper presents a methodology to develop a computationally-efficient approximation of a physicsbased model of underwater acoustic propagation to address this need. The methodology consists of four steps. First, the physics-based model is used to produce a detailed propagation loss data map for the target area. Second, the data is smoothed in preparation for statistical analysis. Third, regression techniques are used to obtain an approximation of the propagation loss model from the smoothed dataset. Finally, additional terms are added to account for the effect of wave motion and random noises. The result is a function that returns the propagation loss of a transmission path based on a small set of parameters pertinent to the locations of the sender and receiver and the transmission frequency.

The proposed methodology was applied to the MontereyMiami Parabolic Equation model (MMPE) as a test case in a study by Diaz-Gonzalez [14]. This study investigated the utility of statistical approximation of the point-to-point propagation results of the MMPE for a simple contour example. By incorporating randomness and wave motion to the approximation, a non-static propagation loss calculation was included in an OPNET [15] implementation of the resultant approximation without inducing a significant performance penalty. The initial propagation data loss map showed that the transmission loss fluctuates heavily over small changes (e.g., $0.5 \mathrm{~m}$ ) to the receiver's vertical and horizontal coordinates, which confirms that the wave motion may have a large temporal impact on acoustic propagation. 
The remainder of the paper describes the approximation technique developed and the authors' implementation of it using OPNET. The paper is organized as follows: Section II explores the complexity of modeling location dependent propagation loss and discusses the need to incorporate environmental factors such as background noise and wave motion; Section III describes the method used to approximate the results of the MMPE; Section IV provides insight into how the approximation was incorporated in an OPNET simulation; and Section V provides conclusions and recommendations for further study.

\section{Methodology}

At the heart of the creation of a realistic and simulationfriendly physical layer model for underwater acoustic communication is the development of an efficient method for predicting the path loss of an acoustic signal. Formally stated, an efficient method is required to compute the function, $P L(A, B, f, t)$, which represents the path loss of an acoustic signal transmitted from node $A$ to node $B$ at time, $t$ and frequency, $f$.

As discussed in the previous section, propagation models created by physicists are typically designed to compute path losses of an acoustic signal iteratively, tracing all paths taken by the signal starting from the source point. This approach, in fact, underscores of the difficulty of deriving a closed form expression of $P L(A, B, f, t)$ that is applicable to all physical channel environments. To be both computationally efficient and accurate, the $P L(A, B, f, t)$ function can only be estimated based on environment-specific parameters, including the ocean bottom contour and the sound speed profile, taking different closed form expressions for different environments.

Since accurate environment-specific path loss data samples

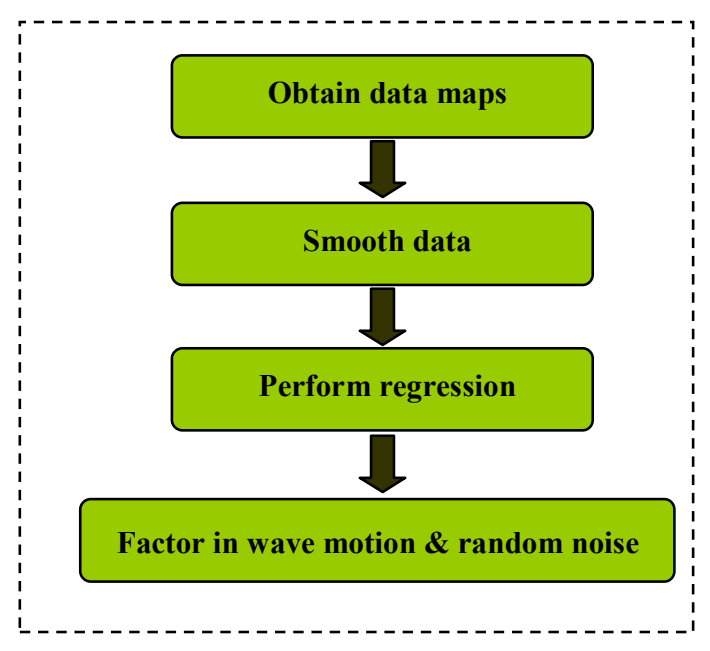

Figure 2: Steps of proposed methodology for an entire area can be obtained using one of the iterative models, given any signal source location and any frequency, a statistical method such as nonlinear regression is a good fit for estimating $P L(A, B, f, t)$. Specifically, we propose a four-step general methodology for estimating $P L(A, B, f, t)$ based on an iterative model, as illustrated in Figure 2.

First, the iterative model is used to produce detailed propagation loss data maps for the target area for various source locations and frequencies of interest. Second, the data is smoothed in preparation for statistical analysis. Our initial experience from performing regression over datasets produced by the MMPE model indicated strongly the need to smooth the data in order for the regression to produce a good fit [14]. Third, regression techniques are used to obtain a time independent approximation of the propagation loss model from the smoothed dataset. Finally, a time variable is factored into the approximation to account for the effect of wave motion and random noise. The result shall be a function with a closed form expression, which returns the propagation loss of a transmission path based on the values of a small set of variables pertinent to the locations of the sender and receiver and the transmission frequency.

\section{ApProximating the MMPE Propagation MODEL}

We have applied the four-step statistical approximation methodology described in the previous section to the MMPE model and successfully obtained an estimated PL function that is environment specific. The results are presented in this section.

\section{A. Obtain Data}

We used an existing FORTRAN based MMPE software package [13] to obtain the data samples. For brevity, the details of installing and running the MMPE software are omitted. The software takes several input files where environment-specific parameters, including source depth, bottom profile, range, sound speed profile, and signal frequency, may be specified. For this study, the input files were configured for a hypothetical area of one kilometer in range and 250 meters depth with a gradient of 50 meters per km, as illustrated in Figure 3(b). The sound speed was set to be constant everywhere. Each execution of the software computes a PL data map for a slice (radial) of the water column as shown in Figure 3(a) for a given signal source depth, $d_{A}$ and a given signal frequency, $f$. The resolution of the data map was set to $2000 \times 500$, corresponding to the "coarse" mode of the MMPE model. For this study, the source depth was varied 7 times from 5 to 35 meters at 5 meter intervals, and the signal frequency modeled varied 11 times from $10 \mathrm{kHz}$ to $20 \mathrm{KHz}$, in $1 \mathrm{KHz}$ increments. In other words, a total of 77 data maps were collected for all the different source depth and signal frequency combinations. These data maps were then merged into one single data file to be used as input to a regression software package. 


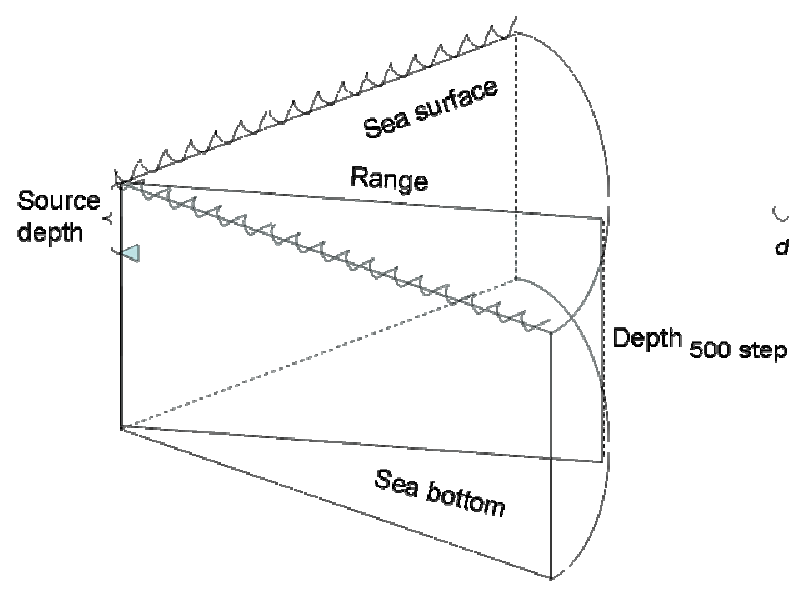

(a)

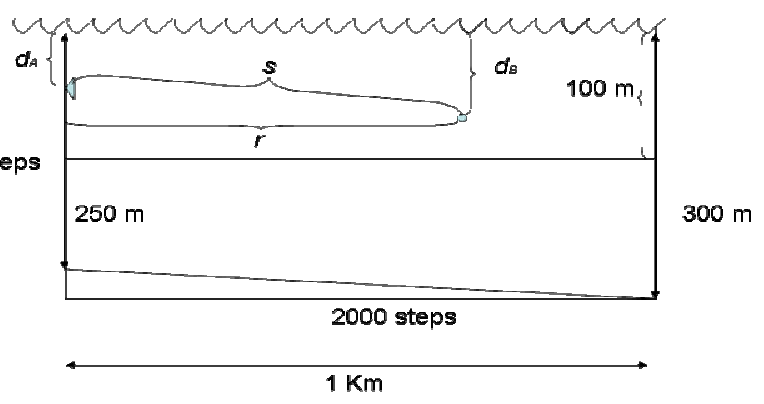

(b)

Figure 3: Area definition of MMPE model

\section{B. Smooth Data}

Initially, we tried using the Microsoft Excel ${ }^{\mathrm{TM}}$ program for the regression analysis. However, it became quickly apparent Excel $^{\mathrm{TM}}$ cannot handle the size (in terms of rows) of the input file, which totals $77 \times 2000 \times 500=77,000,000$. We then experimented with the S-Plus software (version 7.0 for Windows $^{\mathrm{TM}}$ build 7187) from Insightful Corporation [16]. While the S-Plus software was able to load the data file, the results from numerous regression attempts were not satisfactory, with residual standard errors being $10 \mathrm{~dB}$ or more. This highlights the need to pre-process (smooth) the data samples before a regression analysis. For this study, the data is smoothed in two ways. First, the resolution of each PL data map was reduced by a factor of 5 to $400 \times 100$. The PL value of each data point in the new data map is the average PL value of 25 data points in a $5 \times 5$ grid of the original data map. Second, as illustrated in Figure 4, we observed that the PL values for data points in close ranges (within 50 meters) of the signal source have a very different and more dynamic range than other data points in the same data map. This is due to the shadow effect. Since in most network simulation scenarios communicating nodes are more than 50 meters apart, we removed data points with range within 50 meters of the signal source and focused on estimating a PL function that is accurate for ranges greater than 50 meters. The resulting data maps for 3 of the 11 frequencies are shown in Figure 5.

\section{Perform Regression}

The data produced by the MMPE software can be thought of as sample points of a $\operatorname{PL}\left(d_{A}, d_{B}, r, f\right)$ function, where $d_{A}$ and $d_{B}$ are node $\mathrm{A}$ depth and node $\mathrm{B}$ depth in meters, respectively, and $r$ is the range of B (in meters) from A's point of view. $r$ is really the horizontal distance between $\mathrm{A}$ and $\mathrm{B}$. Since a network simulation typically considers the absolute distance between two nodes rather than their horizontal distance, we transformed the data into sample points of $\operatorname{PL}\left(d_{A}, d_{B}, s, f\right)$, where $s=\sqrt{r^{2}+\left(d_{A}-d_{B}\right)^{2}}$ is the absolute distance between $A$ and $B$. We fed the data to the S-Plus regression software and controlled the search space by leveraging known formulas about different aspects of acoustic propagation [14]. In the end, we
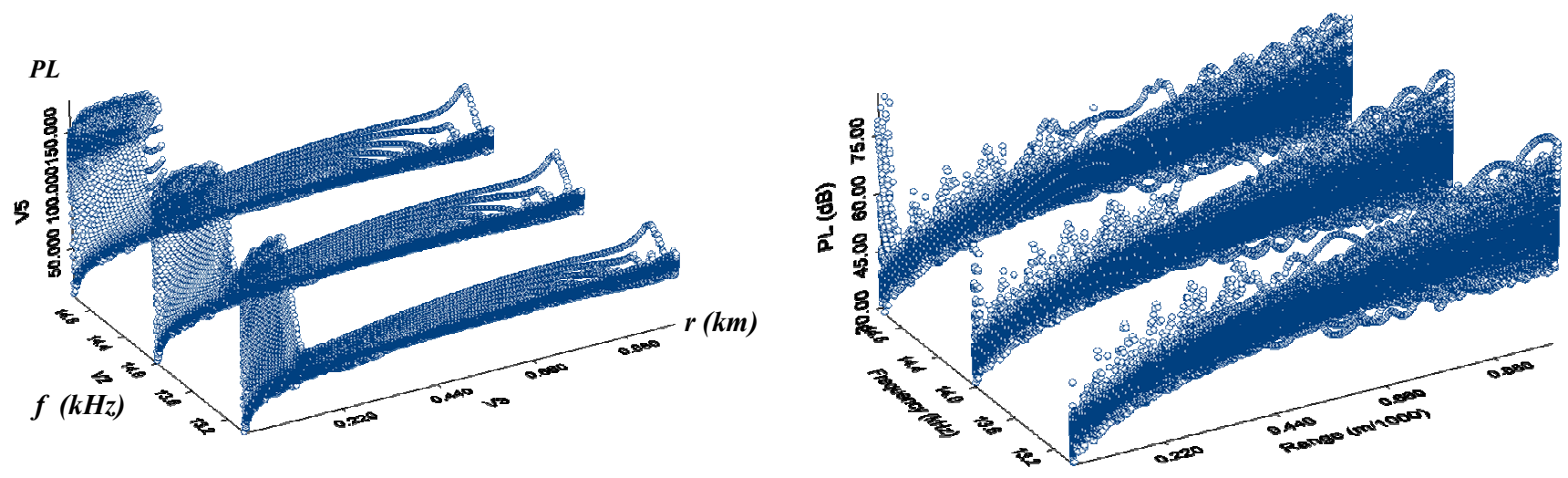

Figure 4. Outliers at short ranges $(<50$ meters $)$

Figure 5: Data ready for regression 
were able to obtain a fit from the regression that has a very reasonable residual standard error of $2.76558 \mathrm{~dB}$. It has the following form:

$$
\begin{aligned}
& P L\left(d_{A}, d_{B}, s, f\right)=\log \left(\frac{(s)^{a 1}\left(d_{A}\right)^{a 2}\left(d_{A}-d_{B}\right)^{a 3}}{a 5\left(s^{*} d_{B}\right)^{a 4}}\right)+ \\
& \quad\left(f^{2}\left(a 6 /\left(1+f^{2}\right)+40 * /\left(4100+f^{2}\right)+.000275\right)+.003\right) *(s / 914)+ \\
& \quad a 7 * d_{B}+a 8 * s
\end{aligned}
$$

Taking a detailed look at the residuals, it can be seen from Figure 6 that they follow a Gaussian distribution with mean of $4.595910 \mathrm{e}-009$ and standard deviation of $2.765339 \mathrm{e}+000$.

\section{Factor in wave motion and random noise}

While analyzing the data samples produced by the MMPE software, we discovered that the PL values of two nearby points (e.g., differed by $0.5 \mathrm{~m}$ in depth range) can be significantly different, sometimes greater than $10 \mathrm{~dB}$. This has motivated us to consider the effect of wave motion on the path loss of an acoustic signal transmission since wave motion will certainly cause the depths of A and B and distance between A and B to fluctuate in the range of 0.5 meter or higher.

Clearly, the effect of wave motion on path loss is time dependent and potentially cyclic. Denote $w(t)$ to be the path loss variation caused by wave motion at time $t$. We have built a simple wave movement model for estimating $w(t)$, based on the observation that under wave motion a water particle will oscillate around its location in a sinusoidal fashion [17]. That movement is represented as circular oscillations that reduce in radius as the depth of the particle increases. The length of that radius is dependent on the energy of the wave and is related to the wave height, as shown in Figure 7. Common waves are one hundred meters in wavelength and have an effect at depths up to 50 meters [17]. For brevity, we omit the details of the $w(t)$ estimation process. They can be found in [14].

The final step was to incorporate a random term, denoted by $e()$, to account for the effect of background noise. We follow the work of [9] and [6] and model the noise with a Gaussian distribution with a maximum value of $20 \mathrm{~dB}$ at the furthest

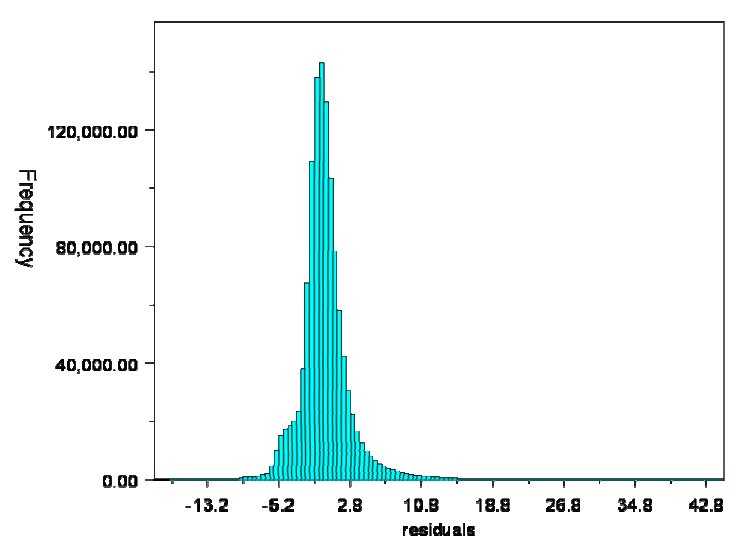

Figure 6: Residual histogram

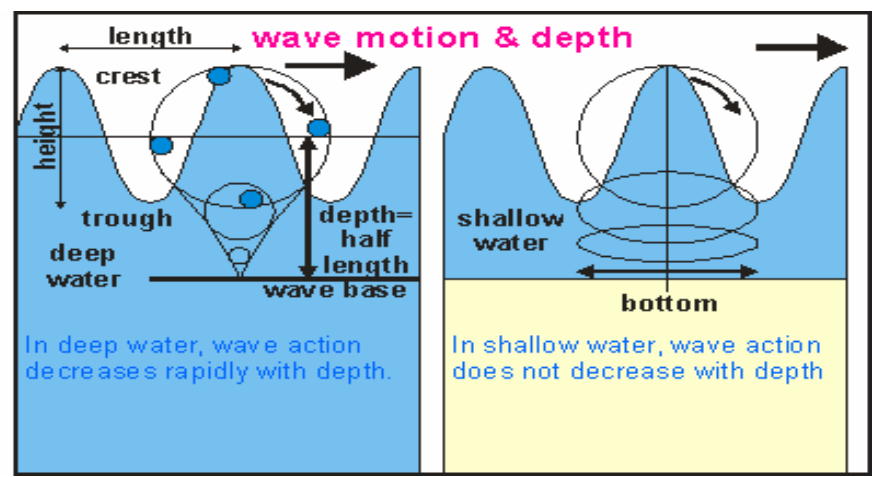

Figure 7: Particle wave movement [17]

feasible distance. Specifically, $e(s)=20\left(\min \left(s / s_{\max }, 1\right)\right) R_{N}$, where $s_{\max }$ is an environment specific parameter representing the likely maximum distance over which $\mathrm{A}$ and $\mathrm{B}$ can communicate, and $R_{N}$ is a random value drawn from a Gaussian distribution with 0 mean and variance of 1 .

Combining results from all the steps and denoting the time invariant function derived from regression by $m\left(d_{A}, d_{B}, s, f\right)$, we obtained the following closed form expression for the path loss:

$$
P L\left(d_{A}, d_{B}, s, f, t\right)=m\left(d_{A}, d_{B}, s, f\right)+w(t)+e(s)
$$

\section{Utility OF THE DERIVED MODEL}

With a computationally feasible approximation in-hand, its utility can be assessed by extending the approximation to an entire network. The authors reconstructed Coelho's simulation using an approximation of the MMPE model, as described in Section 3, to determine whether the approximation generated any significant differences in outcomes. The implementation used a single approximation to represent every potential source. This was a conscious decision, as the purpose of the study was to propose a method for incorporating more robust physical propagation loss models without being detrimental to the runtime performance of the simulation, even though it artificially restricts the fidelity of the approximation for specific sources. Reconstruction of a previous simulation served to verify the concept. While the decision to limit the link approximations to a single regression equation results in artificiality in the topology contour representation, the method can be extended to encompass multiple approximations, each based on a different node pair contour definition. Such an approach would require a more significant up-front effort, as well as more complexity to the initialization of the underlying process model, as a particular approximation equation would need to be selected for each node pair. It is not expected that such complexity would adversely impact the performance of the model during execution as the calculation of each propagation loss would require no more complexity than the model presented here as each loss calculation becomes a simple table look-up as described below. Further simulation and analysis is 


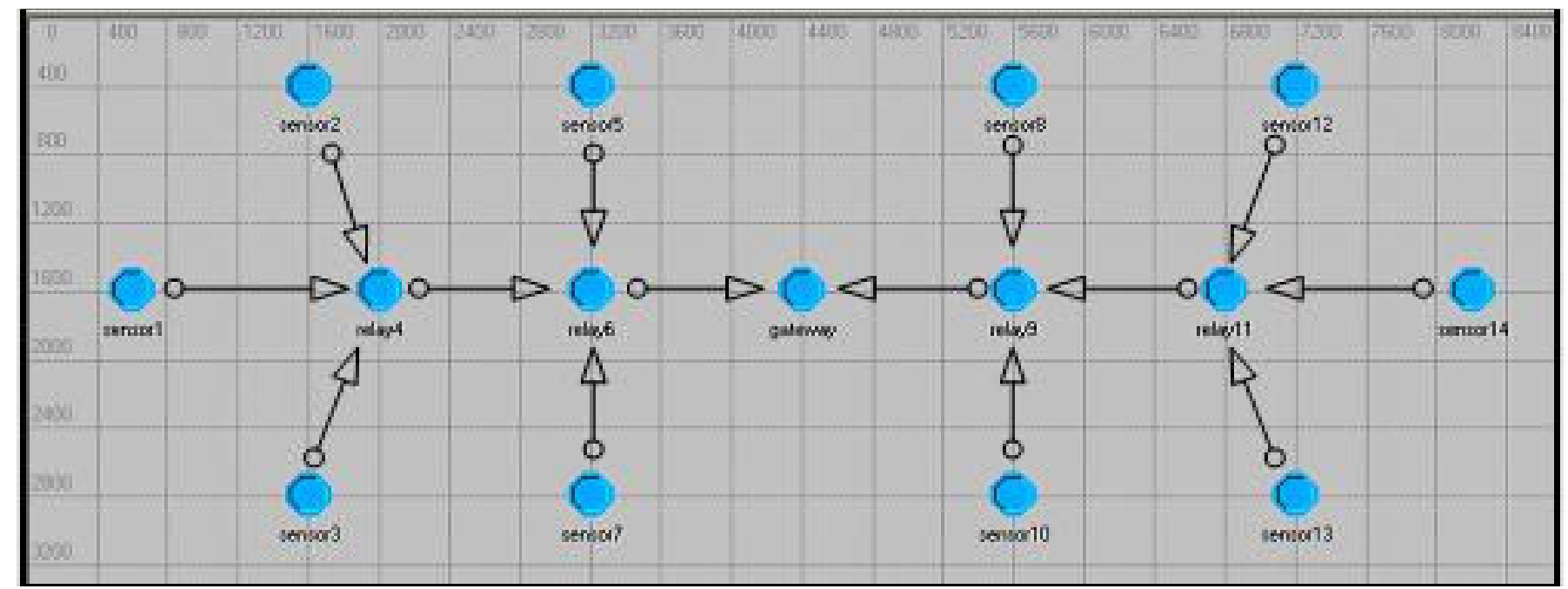

Figure 8: Simulated Network Topology

planned as a separate effort to readdress the results of Coehlo's simulation given this consideration.

No change was made to the original OPNET implementation other than to introduce the calculation of the estimated propagation loss in the physical layer process and a revision of the collision detection scheme at the MAC layer to incorporate the projected loss values. Traffic loading for the simulation included periodic background traffic introduced by leaf nodes, representing sensor data typical of underwater acoustic sensor networks, and exponentially distributed traffic to represent ad hoc messages injected into the network. All traffic flows to a "gateway" which collects statistics on received traffic then drops received packets. The gateway is typical of UANs which communicate with external entities through a special node equipped with both acoustic and RF systems. Retained is the assumption that the acoustic channel varies sufficiently slow over time such that the channel may be considered static over the reception of one message. This assumption is consistent with those of [5-7].

\section{A. OPNET Implementation}

The network modeling and simulation tool, OPNET Modeler, Educational Version, Release 10.5.A PL3, Build 2570, was chosen to implement the models of the network under study. Basic modeling in OPNET typically involves three stages: network model specification, node composition, and process model development. Network specification is the stage where the general network topology is defined (size, technologies, nodes, links, etc.). Node composition establishes the behavior of each network object defined in the network. The process model defines the underlying functionality of each one of the modules defined in the node model. A process model is represented by a Finite State Machine (FSM). Functionality for each process model is implemented by C-code specified by user when he constructs the corresponding FSM.

A tree topology, shown in Figure 8, was considered where the leaf nodes, corresponding to sensor nodes, generate traffic and core relay nodes forward received traffic toward the gateway. The simulation considered two MAC mechanisms, collision avoidance and an Aloha derivative. A fundamental difference between the Aloha-derivative and the collision avoidance schemes is whether or not the sender defers transmission to participate in a reservation mechanism (RTSCTS exchange or reservation expiration). Thus, for the Alohaderivative the defer state is simply a pass-through, while for the collision avoidance model it defines the underlying carrier sense and RTS-CTS exchange. This difference is implemented in the MAC layer process and is the same for both the original analysis and this analysis. Collision detection, and corresponding packet discard are accomplished by the receiving nodes MAC layer based o the propagation loss value calculated by the sender and tagged to the frame. The key difference between the two simulations is the implementation of the Physical Layer, depicted in Fig. 9.

For the original simulation the sending node determined the recipients based on a simple range calculation. The modified simulation bases this decision on the value derived from the MMPE approximation, tagging a loss value to each frame. Only those frames whose loss value is below the pre-defined

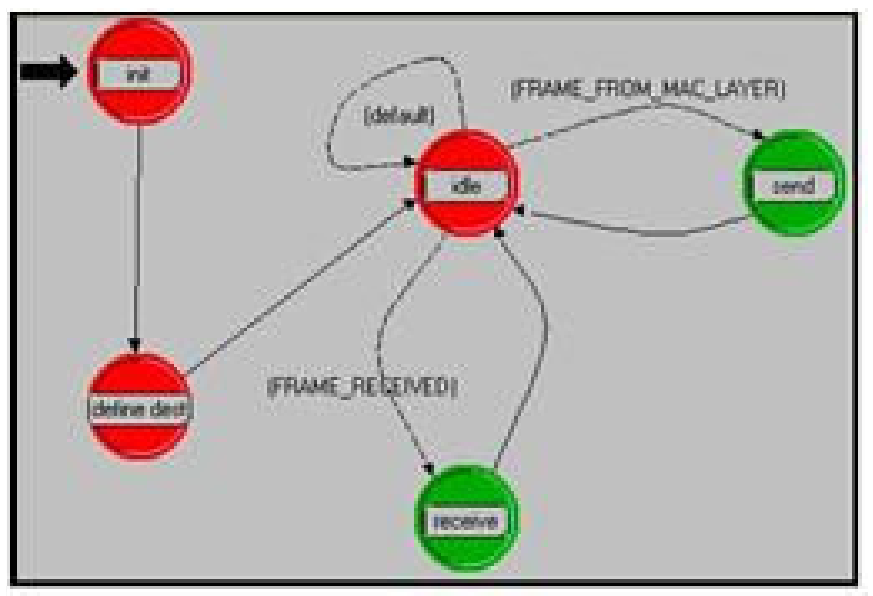

Figure 9: Physical Layer Process Model 
acceptance threshold are forwarded to the respective recipient. The incorporation of wave motion was placed in the simulation initialization to minimize execution time during the simulation run. This was done by defining eight wave motion states and calculating the expected effect for each state. These values were then placed in a table to be referenced based on the time a message is generated. This was a deliberate trade-off between execution complexity and fidelity of the wave motion approximation. The performance of the model, both in terms of run-time execution and the MAC protocol results produced by the two propagation loss models, as implemented in the two different simulations, is discussed in the next subsection.

\section{B. Experience from Initial Simulation Experiments}

The goal of this effort was to determine whether or not a more robust propagation model, based on high fidelity acoustic engineering models, could be incorporated in an off-the-shelf discrete event simulation to provide more robust loss projections for UAN simulation. A comparison of the results of each simulation model was done to determine whether the change in the propagation loss model induced any unexpected changes in the outcome of the simulation. The comparison of the performance of the two MAC protocols by each of the simulations was based on two metrics, the average end-to-end delay of network traffic and the network throughput. The throughput was a measure of the amount of data delivered to the gateway during the course of the simulation. The level of traffic introduced required a significant number of propagation loss calculations as the arrival of each message at each neighbor node had to be calculated such that any collision could be detected as well as the likelihood of receipt be each neighbor.

The primary concern was whether the incorporation of the more robust propagation loss model would result in substantial performance degradation, such that the resulting simulation would not be responsive. Such was not the case, as the typical simulation run, with the loss approximation took approximately 3.5 seconds to execute on a 64-bit AMD 3700+ processor-based laptop computer with $2 \mathrm{~GB}$ of RAM. By contrast, the execution of a single run of the MMPE model took approximately 20 minutes. Each simulation run included calculating the propagation loss for each message to every other node to ensure capture of any collision event, further demonstrating the computational advantage of the approximation equation. This performance suggests the enhanced model is very suitable for UAN performance modeling.

The results of the enhanced model were in general agreement with the earlier model with respect to the relative performance of the two MAC protocols under consideration. However, the inclusion of randomness due to background noise and wave motion resulted in fluctuations in the reception of messages between neighboring nodes and calls into question the assumption that links are temporally bi-directionally stable. The fluctuations resulted in failures in the exchange of RTSCTS messages for the collision avoidance scheme which adversely impacted the throughput achieved for that scheme. These fluctuations should be validated against data gathered from real world experimentation to determine if they are consistent with that data. The respective performance of the two protocols is anecdotally discussed below.

Figures 10 and 11 show the modeled performance of the two protocols by the each of the simulations. In particular, the top line in Fig. 10 represents the combined periodic and nonperiodic traffic for the Aloha-derived protocol and the line below it is the composite traffic for the collision avoidance scheme, both modeled with the simple range calculation to determine message reception. The vertical axis represents throughput in bits per second. While the Aloha-derived protocol appears to out perform the other, the two protocols perform quite similar, but as loads marginally increase the Aloha protocol performance is better, confirming the authors' suspicion [8]. Interestingly, when a more robust propagation calculation is used, in this case the approximation of the MMPE calculation as shown in Figure 11, the trend seems to be consistent, as the top line is that of the Aloha protocol, although the overall throughput for both protocols dropped significantly - close to one order of magnitude lower. Again, the only difference between the two models was the implementation of the loss (reception) determination.

The results strongly suggest that the difference in propagation loss fidelity can have a significant impact on the simulated performance of the network under study. When the performance of a particular network deployment is of concern it is reasonable to incorporate environmental factors such as topology contours into the loss prediction model which can influence the performance of the fielded system. The methodology presented above demonstrates the utility of providing statistical approximations to more complex propagation loss models such that the inclusion of these loss calculations does not significantly impact the performance of the simulation itself.

\section{CONCLUDING REMARKS}

Even though the proposed statistical methodology is conceptually sound, strictly speaking its effectiveness has not been validated. Another area that merits further investigation is the modeling of wave motion. No doubt we have only scratched the surface of this line of research. Nevertheless, we would like to bring the attention of underwater networking protocol designers to the rich set of acoustic engineering models developed by physicists and the potential of utilizing these models to create a high fidelity physical layer for computer simulations. The potential benefits are certainly worthy the effort.

\section{ACKNOWLEDGMENT}

The authors appreciate the support of Kevin Smith in making the MMPE model available and providing guidance with respect to its use. Appreciation is also extended to Wen-Bin Yang for providing thoughtful feedback on this paper. 


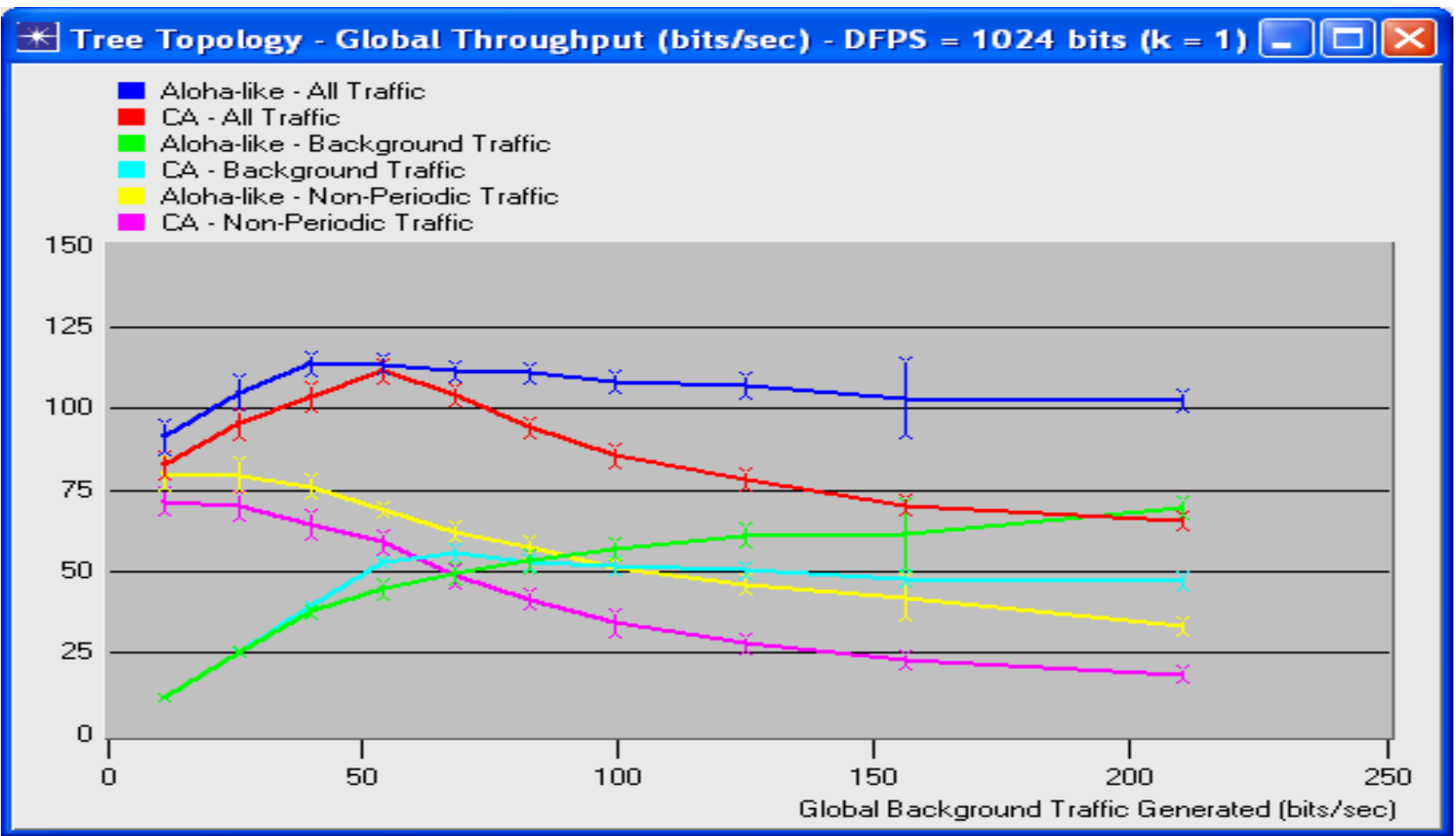

Figure10: Throughput: Reception based on range

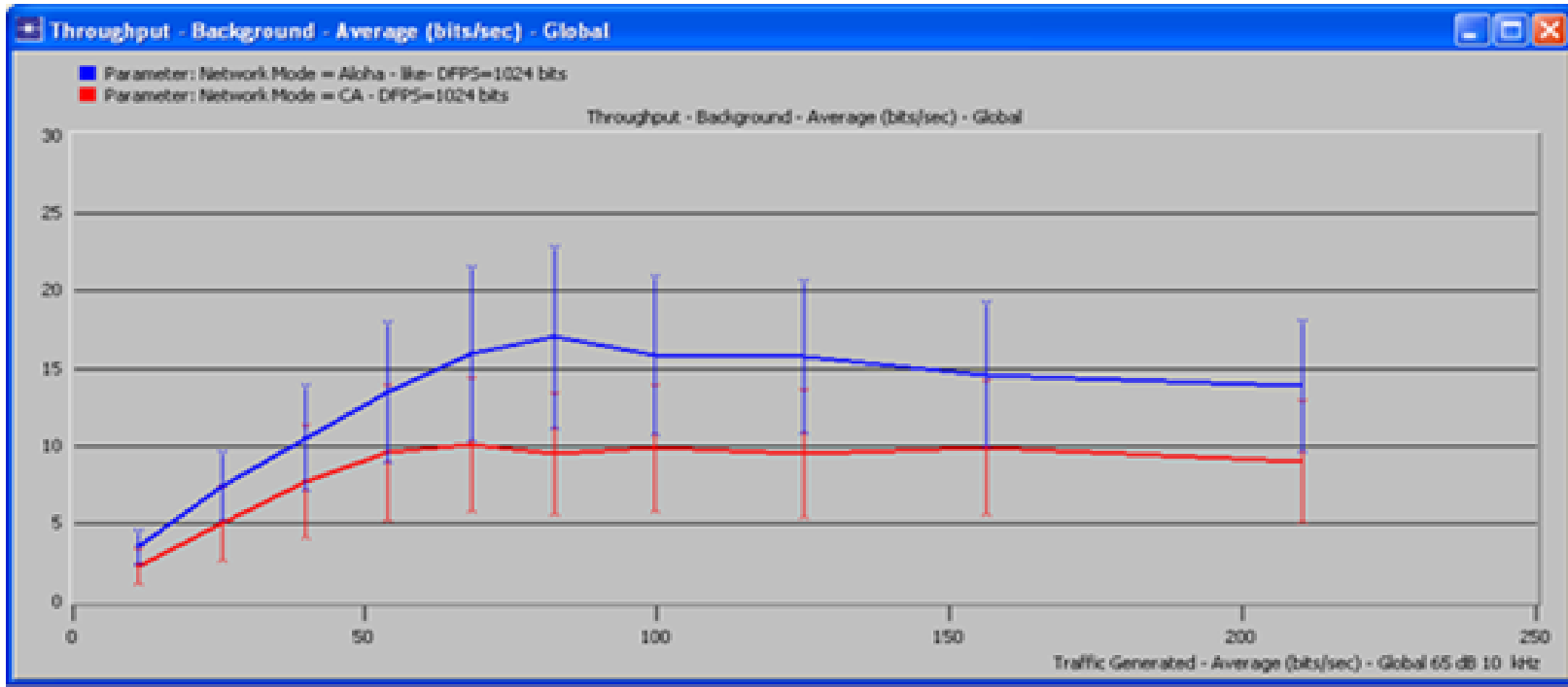

Figure 11: Throughput: Reception based on MMPE approximation 


\section{REFERENCES}

[1] J. Rice, "SEAWEB Brief Protocol," The Technical Cooperative Program Initiative in Undersea Networking Letter \#8, Jun21, 2006, pg 9

[2] G. Hartfield. "Link-Layer and Network-Layer Performance of an Undersea Acoustic Network at Fleet Battle Experiment - India”, Master's Thesis (MS-ISO), Naval Postgraduate School, June 2003

[3] Xie, Geoffrey and Gibson, John, "A Network Layer Protocol for UANs to Address Propagation Delay Induced Performance Limitations," Proceedings of the MTS/IEEE Oceans Conference, November 2001

[4] M. Stojanovic, L. Freitag, J. Leonard and P. Newman, "A Network Protocol for Multiple AUV Localization," in Proc. IEEE OCEANS 2002, vol. 1, pp. 604-611, 2002

[5] F. Salva-Garau, M. Stojanovic, "Multi-cluster protocol for ad hoc mobile underwater acoustic networks" in Proc IEEE OEANS 2003, vol. 1, pp. 9198

[6] Sozer, Ethem M., Stojanovic, Milica and Proakis, John G., "Design and Simulation of an Underwater Acoustic Local Area Network," Northeastern University, Communications and Digital Signal Processing Center, 409 Dana Research Building, Boston, Massachusetts, 1999.

[7] Coelho, Jose, "Underwater Acoustic Networks: Evaluation of the Impact of Media Access Control on Latency, in a Delay Constrained Network," Master's Thesis (MS-CS), Naval Postgraduate School, Monterey, California, March 2005.

[8] Gibson, John, Xie, Geoffrey, Coelho, José and Diaz-Gonzalez, "The Impact of Contention Resolution verses a priori Channel Allocation Delay Constrained Network," Undersea Networking (Unet) Workshop, in support of The Technical Cooperative Program (TTCP), Monerey, CA, January 2005.

[9] Urick, Robert J., "Principles of Underwater Sound," 3rd Edition, Peninsula Publishing, 1983.

[10] Brekhovskikh, L. and Lysanov, Yu, "Fundamentals of Ocean Acoustics," Springer-Verlag Berlin Heidelberg New York, 1982.

[11] R.A. Zingarelli and D.B. King, "RAM to Navy Standard Parabolic Equation: Transition from Research to Fleet Acoustic Model," 2003 NRL Review, NRL website: http://www.nrl.navy.mil/content.php?P=03REVIEW212]

[12] Smith, Kevin B., "Convergence, Stability, and Variability of Shallow Water Acoustic Predictions Using a Split-Step Fourier Parabolic Equation Model,” J. Comp. Acoust., Vol. 9, No. 1, pp. 243-285, 2001 - Special Issue of Proceedings of the Shallow Water Acoustic Modeling (SWAM'99) Workshop, 8 - 10 September 1999 (eds. Alex Tolstoy and Kevin B. Smith)

[13] Smith, Kevin B. and Tappert, Frederick, "Monterrey-Miami Parabolic Equation," Release Documentation.

[14] Diaz-Gonzalez, "Underwater Acoustic Networks: An Acoustic Propagation Model for Simulation of Underwater Acoustic Networks," Master's Thesis (MS-CS), Naval Postgraduate School, Monterey, California, December 2005.

[15] OpNet Version 11.0, "Wireless Manual: Radio Transceiver Pipeline," Modeler, Documentation, OPNET Technologies, and Inc., 2003.

[16] S-Plus Version 7.0, Help Documentation, Insightful Corp. 2005.

[17] Van Dorn W..," Oceanography and seamanship", Dodd, Mead, New York, 1974 